International Journal of Pure and Applied Mathematics

Volume 106 No. 3 2016, 791-799

ISSN: 1311-8080 (printed version); ISSN: 1314-3395 (on-line version)

url: http://www.ijpam.eu

doi: 10.12732/ijpam.v106i3.8

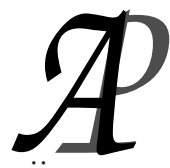

\title{
COMMON COUPLED FIXED POINT THEOREMS OF TWO MAPPINGS SATISFYING GENERALIZED CONTRACTIVE CONDITION IN CONE METRIC SPACE
}

\author{
H.K. Nashie ${ }^{1}$, Yumanm Rohen ${ }^{2}$, Chhatrajit Thokchom ${ }^{3} \S$ \\ ${ }^{1}$ Disha Institute of Management and Technology \\ Raipur, INDIA \\ ${ }^{2,3}$ Department of Basic Sciences and Humanities \\ NIT Manipur, Imphal,795001, INDIA
}

\begin{abstract}
In this paper, we prove the existence and uniqueness of a common coupled fixed point theorem in complete cone metric space for a pair of mappings satisfying a generalised contractive condition. Some results are also given in the form of corollaries. The results are also verified with the help of example.
\end{abstract}

AMS Subject Classification: $47 \mathrm{H} 10,54 \mathrm{H} 25$

Key Words: cone metric space, mixed monotone property, coupled fixed point, fixed point theorems

\section{Introduction}

Dajun Guo and V. Lakshmikantham [1] gave the existence theorems of the coupled fixed points for both continuous and discontinuous operators and gave applications to the initial value problems of ordinary differential equations with discontinuous right-hand sides. Bhaskar and Lakshmikantham [2] proved the

Received: September 16, 2015

Published: February 27, 2016

$\S^{\S}$ Correspondence author
(C) 2016 Academic Publications, Ltd.

url: www.acadpubl.eu 
existence of coupled fixed point theorem for a mixed monotone mapping in a metric space with the help of partial order, using a weak contractivity type of assumption. Since then this new concept is extended and used in various directions. This concept is extended to tripled fixed point by Berinde and Borcut [3] to quadrupled fixed point by Karapinar [4]. Coupled fixed point is also extended in various spaces like metric space, G-metric space, b-metric space, partially ordered metric space, fuzzy metric space, cone metric space etc.

The concept of cone metric space introduced by Huang and Zang [5] in 2007 as generalizations of metric space. They generalised metric space by replacing the set of real numbers with an ordering Banach space. Thus, cone naturally induces a partial order in Banach spaces. In recent years many authors established various coupled fixed point theorems in cone metric space (see [6-17] and references there in).

Let $E$ be a real Banach Space. A subset $P$ of $E$ is called a cone if

1. $P$ is closed, non-empty and $P \neq 0$

2. $a, b \in \mathbb{R}, a, b \geq 0$ and $x, y \in P$ imply $a x+b y \in P$

3. $P \cap(-P)=\{0\}$.

Given a cone $P \subset E$ we define the partial ordering $\leq$ with respect to $P$ by $x \leq y$ if and only if $y-x \in P$. We write $x<y$ to denote that $x \leq y$ but $x \neq y$, while $x<<y$ will stand for $y-x \in$ int.P(interior of $P$ ).

There are two kinds of cone. They are normal cone and non-normal cones. A cone $P \subset E$ is normal if there is a number $K>0$ such that for all $x, y \in P$, $0 \leq x \leq y \Rightarrow\|x\| \leq K\|y\|$. In other words if $x_{n} \leq y_{n} \leq z_{n}$ and $\lim _{n \rightarrow \infty} x_{n}=$ $\lim _{n \rightarrow \infty} z_{n}=x$ imply $\lim _{n \rightarrow \infty} y_{n}=x$. Also, a cone $P \subset E$ is regular if every increasing sequence which is bounded above is convergent.

The aim of the present work is to prove the existence and uniqueness of a common coupled fixed point theorem satisfying a generalised contractive condition. Some other results are also given in the form of corollaries.

Definition 1.1. ([5]) Let $X$ be a nonempty set. Suppose the mapping $d: X \times X \rightarrow E$ satisfies the following conditions:

1. $0<d(x, y)$ for all $x, y \in X$ and $d(x, y)=0$ iff $x=y$.

2. $d(x, y)=d(y, x)$ for all $x, y \in X$.

3. $d(x, y) \leq d(x, z)+d(z, y)$ for all $x, y, z \in X$.

Then $d$ is called a cone metric on $X$ and $(X, d)$ is called a cone metric space. 
Definition 1.2. ([5]) Let $(X, d)$ be a cone metric space(CMS), $x_{n}$ a sequence in $X$ and $x \in X$. For every $c \in E$ with $0 \ll c$, we say that $x_{n}$ is

1. a Cauchy sequence if there is some $k \in \mathbb{N}$ such that, for all $n, m \geq$ $k, d\left(x_{n}, x_{m}\right) \ll c ;$

2. a convergent sequence if there is some $k \in \mathbb{N}$ such that, $n \geq k, d\left(x_{n}, x\right) \ll c$. Then $x$ is called limit of the sequence $x_{n}$.

3. $(X, d)$ is a complete cone metric space if every Cauchy sequence in $X$ is convergent in $X$.

Let $(X, d)$ be a cone metric space(CMS). Then the following properties are often used :

1. If $E$ is a real Banach space with a cone $P$ and if $a \preceq h a$ where $a \in P$ and $h \in[0,1)$, then $a=0$.

2. If $\theta \preceq u \ll c$ for each $\theta \ll c$, then $u=\theta$.

3. If $a \preceq b+c$ for each $\theta \ll c$, then $a \preceq b$.

4. If $u \preceq v$ and $v \preceq w$, then $u \ll w$.

5. If $c \in$ int.P, $0 \preceq a_{n}$ and $a_{n} \rightarrow \theta$, then there exists an $k$ such that for all $n>k$ we have $a_{n} \ll c$.

It follows from (e) that the sequence $x_{n}$ converges to $x \in X$ if $d\left(x_{n}, x\right) \rightarrow \theta$ as $n \rightarrow \infty$ and $x_{n}$ is a Cauchy sequence if $d\left(x_{n}, x_{m}\right) \rightarrow \theta$ as $s, m \rightarrow \infty$. In the case when the cone is not necessarily normal, then $d\left(x_{n}, y_{n}\right) \rightarrow d(x, y)$ if $x_{n} \rightarrow x$ and $y_{n} \rightarrow y$ is not applicable.

Definition 1.3. ([2]) An element $(x, y) \in X \times X$ is called a coupled fixed point of the mapping $F: X \times X \rightarrow X$ if $x=F(x, y)$ and $y=F(y, x)$.

\section{Main Results}

We prove the following theorem:

Theorem 2.1. Let $(X, d)$ be a complete cone metric space with a cone $P$ having non-empty interior and let $S, T: X \times X \rightarrow X$ satisfying

$$
d(S(x, y), T(u, v)) \leq a_{1} d(x, u)+a_{2} d(S(x, y), x)+a_{3} d((y, v)
$$




$$
+a_{4} d(T(u, v), u)+a_{5} d(S(x, y), u)+a_{6} d(T(u, v), x),
$$

for all $x, y, u, v \in X$, where $a_{i}, i=1,2, \ldots 6$ are non-negative real numbers such that $a_{1}+a_{2}+a_{3}+a_{4}+a_{5}+2 a_{6}<1$.

Then $S$ and $T$ have a unique common coupled fixed point in $X$.

Proof. Let $x_{0}$ and $y_{0}$ be arbitrary points in $X$. Let

$$
x_{2 k+1}=S\left(x_{2 k}, y_{2 k}\right), y_{2 k+1}=S\left(y_{2 k}, x_{2 k}\right)
$$

and

$$
x_{2 k+2}=T\left(x_{2 k+1}, y_{2 k+1}\right), y_{2 k+2}=T\left(y_{2 k+1}, x_{2 k+1}\right)
$$

for $k=0,1,2, \ldots$.

Then

$$
\begin{aligned}
& d\left(x_{2 k+1}, x_{2 k+2}\right)= d\left(S\left(x_{2 k}, y_{2 k}\right), T\left(x_{2 k+1}, y_{2 k+1}\right)\right) \\
& \leq a_{1} d\left(x_{2 k}, x_{2 k+1}\right)+a_{2} d\left(S\left(x_{2 k}, y_{2 k}\right), x_{2 k}\right)+a_{3} d\left(y_{2 k}, y_{2 k+1}\right) \\
&+a_{4} d\left(T\left(x_{2 k+1}, y_{2 k+1}\right), x_{2 k+1}\right)+a_{5} d\left(S\left(x_{2 k}, y_{2 k}\right), x_{2 k+1}\right) \\
&+a_{6} d\left(T\left(x_{2 k+1}, y_{2 k+1}\right), x_{2 k}\right) \\
&= a_{1} d\left(x_{2 k}, x_{2 k+1}\right)+a_{2} d\left(x_{2 k+1}, x_{2 k}\right)+a_{3} d\left(y_{2 k}, y_{2 k+1}\right) \\
&+a_{4} d\left(x_{2 k+2}, x_{2 k+1}\right)+a_{5} d\left(x_{2 k+1}, x_{2 k+1}\right)+a_{6} d\left(x_{2 k+2}, x_{2 k}\right) \\
&= a_{1} d\left(x_{2 k}, x_{2 k+1}\right)+a_{2} d\left(x_{2 k+1}, x_{2 k}\right)+a_{3} d\left(y_{2 k}, y_{2 k+1}\right) \\
&+a_{4} d\left(x_{2 k+1}, x_{2 k+2}\right)+a_{5} \times 0 \\
&+a_{6}\left\{d\left(x_{2 k}, x_{2 k+1}\right)+d\left(x_{2 k+1}, x_{2 k+2}\right)\right\} \\
& \Rightarrow\left(1-a_{4}-a_{6}\right) d\left(x_{2 k+1}, x_{2 k+2}\right) \leq\left(a_{1}+a_{2}+a_{6}\right) d\left(x_{2 k}, x_{2 k+1}\right)+a_{3} d\left(y_{2 k}, y_{2 k+1}\right) \\
& \Rightarrow d\left(x_{2 k+1}, x_{2 k+2}\right) \leq \frac{a_{1}+a_{2}+a_{6}}{1-a_{4}-a_{6}} d\left(x_{2 k}, x_{2 k+1}\right)+\frac{a_{3}}{1-a_{4}-a_{6}} d\left(y_{2 k}, y_{2 k+1}\right) .
\end{aligned}
$$

Similarly

$$
\begin{aligned}
d\left(y_{2 k+1}, y_{2 k+2}\right) \leq & d\left(S\left(y_{2 k}, x_{2 k}\right), T\left(y_{2 k+1}, x_{2 k+1}\right)\right) \\
\leq & a_{1} d\left(y_{2 k}, y_{2 k+1}\right)+a_{2} d\left(S\left(y_{2 k}, x_{2 k}\right), y_{2 k}\right)+a_{3} d\left(x_{2 k}, x_{2 k+1}\right) \\
& +a_{4} d\left(T\left(y_{2 k+1}, x_{2 k+1}\right), y_{2 k+1}\right)+a_{5} d\left(S\left(y_{2 k}, x_{2 k}\right), y_{2 k+1}\right) \\
& +a_{6} d\left(T\left(y_{2 k+1}, x_{2 k+1}\right), y_{2 k}\right) \\
d\left(y_{2 k+1}, y_{2 k+2}\right) \leq & \frac{a_{1}+a_{2}+a_{6}}{1-a_{4}-a_{6}} d\left(y_{2 k}, y_{2 k+1}\right)+\frac{a_{3}}{1-a_{4}-a_{6}} d\left(x_{2 k}, x_{2 k+1}\right)
\end{aligned}
$$


Adding we have

$$
\begin{aligned}
d\left(x_{2 k+1}, x_{2 k+2}\right)+d\left(y_{2 k+1}, y_{2 k+2}\right) \leq & \frac{a_{1}+a_{2}+a_{3}+a_{6}}{1-a_{4}-a_{6}} d\left(x_{2 k}, x_{2 k+1}\right) \\
& +\frac{a_{1}+a_{2}+a_{3}+a_{6}}{1-a_{4}-a_{6}} d\left(y_{2 k}, y_{2 k+1}\right) \\
\leq & \frac{a_{1}+a_{2}+a_{3}+a_{6}}{1-a_{4}-a_{6}} \\
& \times\left[d\left(x_{2 k}, x_{2 k+1}\right)+d\left(y_{2 k}, y_{2 k+1}\right)\right] \\
= & h\left[d\left(x_{2 k}, x_{2 k+1}\right)+d\left(y_{2 k}, y_{2 k+1}\right)\right],
\end{aligned}
$$

where

$$
0<h=\frac{a_{1}+a_{2}+a_{3}+a_{6}}{1-a_{4}-a_{6}}<1 .
$$

Also, we have

$$
d\left(x_{2 k+2}, x_{2 k+3}\right)+d\left(y_{2 k+2}, y_{2 k+3}\right)=h\left[d\left(x_{2 k+1}, x_{2 k+2}\right)+d\left(y_{2 k+1}, y_{2 k+2}\right)\right]
$$

Therefore

$$
\begin{aligned}
d\left(x_{n}, x_{n+1}\right)+d\left(y_{n}, y_{n+1}\right) \leq h\left[d\left(x_{n-1}, x_{n}\right)+\right. & \left.d\left(y_{n-1}, y_{n}\right)\right] \\
& \leq \cdots \leq h^{n}\left[d\left(x_{0}, x_{1}\right)+d\left(y_{0}, y_{1}\right)\right]
\end{aligned}
$$

Now if $d\left(x_{n}, x_{n+1}\right)+d\left(y_{n}, y_{n+1}\right)=\delta_{0}$ then

$$
\delta_{n} \leq h \delta_{n-1} \leq \cdots \leq h^{n} \delta_{n}
$$

For $m>n$

$$
\begin{aligned}
d\left(x_{n}, x_{m}\right)+d\left(y_{n}, y_{m}\right) & \leq \delta_{m-1}+\delta_{m-2}+\ldots+\delta_{n} \\
& \leq\left(h^{m-1}+h^{m-2}+\ldots+h^{n}\right) \delta_{0} \\
& =h^{n}\left(1+h+\ldots+h^{m-n-1}\right) \delta_{0} \\
& =\frac{h^{n}\left(1-h^{m-n}\right)}{1-h} \delta_{0} \\
& =\frac{h^{n}-h^{m}}{1-h} \delta_{0} \\
& \leq \frac{h^{n}}{1-h} \delta_{0} \rightarrow 0 \text { as } n \rightarrow \infty .
\end{aligned}
$$

From $(e)$ it follow that for $0 \ll c$ and for large $n$, we have $\frac{h^{\mathrm{n}}}{1-h} \delta_{0} \ll c$. 
Thus, according to $(d), d\left(x_{n}, x_{m}\right)+d\left(y_{n}, y_{m}\right) \ll c$. Hence by definition $1.2($ iii $)\left\{d\left(x_{n}, x_{m}\right)+d\left(y_{n}, y_{m}\right)\right\}$ is a Cauchy sequence. Since

$$
d\left(x_{n}, x_{m}\right) \ll d\left(x_{n}, x_{m}\right)+d\left(y_{n}, y_{m}\right)
$$

and

$$
d\left(y_{n}, y_{m}\right) \ll d\left(x_{n}, x_{m}\right)+d\left(y_{n}, y_{m}\right) .
$$

Again by $(d)\left\{x_{n}\right\}$ and $\left\{y_{n}\right\}$ are Cauchy sequences in $X$, so there exists $x$ and $y$ in $X$ such that $x_{n} \rightarrow x$ and $y_{n} \rightarrow y$ as $n \rightarrow \infty$.

Now, we show that $x=S(x, y)$ and $y=S(y, x)$. On the contrary, let us assume that $x \neq S(x, y)$ and $y \neq S(y, x)$ so that $d(x, S(x, y))=k>0$ and $d(y, S(y, x))=l>0$.

Consider

$$
\begin{aligned}
l_{1}=d(x, S(x, y)) \leq & d\left(x, x_{2 k+2}\right)+d\left(x_{2 k+2}, S(x, y)\right) \\
= & d\left(x, x_{2 k+2}\right)+d\left(T\left(x_{2 k+1}, y_{2 k+1}\right), S(x, y)\right) \\
\leq & a_{1} d\left(x, x_{2 k+1}\right)+a_{2} d\left(S(x, y), x_{2 k+1}\right)+a_{3} d\left(y, y_{2 k+1}\right) \\
& +a_{4} d\left(T\left(x_{2 k+1}, y_{2 k+1}\right), x_{2 k+1}\right)+a_{5} d\left(S(x, y), x_{2 k+1}\right) \\
& +a_{6} d\left(T\left(x_{2 k+1}, y_{2 k+1}\right), x\right) \\
= & a_{1} d\left(x, x_{2 k+1}\right)+a_{2} d\left(S(x, y), x_{2 k+1}\right)+a_{3} d\left(y, y_{2 k+1}\right) \\
& +a_{4} d\left(x_{2 k+2}, x_{2 k+1}\right)+a_{5} d\left(S(x, y), x_{2 k+1}\right)+a_{6} d\left(x_{2 k+2}, x\right)
\end{aligned}
$$

(taking $k \rightarrow \infty$ we receive)

$$
\begin{aligned}
= & a_{1} \cdot 0+a_{2} d\left(S(x, y), x_{2 k+1}\right)+a_{3} \cdot 0+a_{4} \cdot 0 \\
& +a_{5} d\left(S(x, y), x_{2 k+1}\right)+a_{6} \cdot 0 \\
& \Rightarrow\left(1-a_{2}-a_{5}\right) d(x, S(x, y)) \leq 0 \\
& \Rightarrow d(x, S(x, y)) \leq 0 \\
& \Rightarrow l_{1} \leq 0
\end{aligned}
$$

which is a contradiction.

Therefore,

$$
d(x, S(x, y))=0 .
$$

So

$$
x=S(x, y) .
$$

Similarly, we can prove that 


$$
y=S((y, x)
$$

It follows, similarly, that

$$
x=T(x, y) \text { and } y=T(y, x) .
$$

So we have proved that $(x, y)$ is a common coupled fixed point of $S$ and $T$.

In order to prove the uniqueness let $\left(x^{\prime}, y^{\prime}\right) \in X \times X$ be another common coupled fixed point of $S$ and $T$.

Then

$$
\begin{aligned}
d\left(x, x^{\prime}\right)= & d\left(S(x, y), T\left(x^{\prime}, y^{\prime}\right)\right) \\
\leq & a_{1} d\left(x, x^{\prime}\right)+a_{2} d(S(x, y), x)+a_{3} d\left(y, y^{\prime}\right) \\
& +a_{4} d\left(T\left(x^{\prime}, y^{\prime}, x^{\prime}\right)\right) \\
= & a_{5} d\left(S(x, y), x^{\prime}\right)+a_{6} d\left(T\left(x^{\prime}, y^{\prime}\right), x\right) \\
= & a_{1} d\left(x, x^{\prime}\right)+a_{2} d(x, x)+a_{3} d\left(y, y^{\prime}\right) \\
& +a_{4} d\left(x^{\prime}, x^{\prime}\right)+a_{5} d\left(x, x^{\prime}\right) \\
& +a_{6} d\left(x^{\prime}, x\right) \\
\Rightarrow & \left(a_{1}+a_{5}+a_{6}\right) d\left(x^{\prime}, x\right)+a_{3} d\left(y, y^{\prime}\right) \\
\Rightarrow\left(1-a_{1}-a_{5}-a_{6}\right) d\left(x, x^{\prime}\right) \leq & a_{3} d\left(y, y^{\prime}\right) \\
\Rightarrow d\left(x, x^{\prime}\right) \leq & \frac{a_{3}}{1-a_{1}-a_{5}-a_{6}} d\left(y, y^{\prime}\right)
\end{aligned}
$$

Similarly, one can prove that

$$
d\left(y, y^{\prime}\right) \leq \frac{a_{3}}{1-a_{1}-a_{5}-a_{6}} d\left(x, x^{\prime}\right)
$$

Adding we get

$$
\begin{aligned}
d\left(x, x^{\prime}\right)+d\left(y, y^{\prime}\right) & \leq \frac{a_{3}}{1-a_{1}-a_{5}-a_{6}} \\
\Rightarrow\left(1-\frac{a_{3}}{1-a_{1}-a_{5}-a_{6}}\right)\left[d\left(x, x^{\prime}\right)+d\left(y, y^{\prime}\right)\right] & \leq 0 \\
\Rightarrow d\left(x, x^{\prime}\right)+d\left(y, y^{\prime}\right) & =0 \\
\Rightarrow x=x^{\prime} \text { and } y=y^{\prime} . &
\end{aligned}
$$


Corollary 2.2. Let $(X, d)$ be a complete cone metric space with a cone $P$ having non-empty interior and let $T: X \times X \rightarrow X$ satisfying

$$
\begin{aligned}
d(T(x, y), T(u, v)) \leq & a_{1} d(x, u)+a_{2} d(T(x, y), x)+a_{3} d(y, v)++a_{4} d(T(u, v), u) \\
& +a_{5} d(T(x, y), u)+a_{6} d(T(u, v), x),
\end{aligned}
$$

for all $x, y, u, v \in X$ where $a_{i}, i=1,2, \ldots, 6$ are non-negative real numbers such that $a_{1}+a_{2}+a_{3}+a_{4}+a_{5}+2 a_{6}<1$. Then $T$ has a unique coupled fixed point in $X$.

Corollary 2.3. Let $(X, d)$ be a complete cone metric space with a cone $P$ having non-empty interior and let $S, T: X \times X \rightarrow X$ satisfying

$$
\begin{aligned}
d(S(x, y), T(u, v)) \leq & a d(x, u)+b d(y, v))+c[d(S(x, y), x)+d(T(u, v), u)] \\
& +e[d(S(x, y), u)+d(T(u, v), x)]
\end{aligned}
$$

for all $x, y, u, v \in X$ where $a, b, c, e$ are non-negative real numbers such that $a+b+2 c+2 e<1$. Then $S$ and $T$ have a unique common coupled fixed point in $X$.

Corollary 2.4. Let $(X, d)$ be a complete cone metric space with a cone $P$ having non-empty interior and let $T: X \times X \rightarrow X$ satisfying

$$
\begin{aligned}
d(T(x, y), T(u, v)) \leq & a d(x, u)+b d(y, v)+c[d(T(x, y), x)+d(T(u, v), u)] \\
& +e[d(T(x, y), u)+d(T(u, v), x)]
\end{aligned}
$$

for all $x, y, u, v \in X$ where $a, b, c, e$ are non-negative real numbers such that $a+b+2 c+2 e<1$. Then $T$ has a unique coupled fixed point in $X$.

Example 2.5. Let $X=\{0,1\}$ and $E=\mathbb{R}$. Define $d: X \times X \rightarrow E$ by $d(x, y)=\frac{2}{3}(x-y)^{2}$ for all $x, y \in X$. Then $(X, d)$ is a cone metric space. Define $S, T: X \times X \rightarrow X$ as follows

$$
\begin{aligned}
S(x, y) & =\frac{x y}{4} \\
T(x, y) & =\frac{x y}{3} .
\end{aligned}
$$

Take $a_{1}=\frac{1}{2}, a_{2}=1=a_{5}, a_{3}=2, a_{4}=0=a_{6}$. We can show that $S$ and $T$ satisfy the generalized contractive condition of Theorem 2.1. We got the point $(0,0)$ is a unique common fixed point of $S$ and $T$. 


\section{References}

[1] D. Guo and V. Lakshmikantham, Coupled Fixed Points of Nonlinear Operators with Applications, Nonlinear Analysis:TMA, 11 (1987) 623-632.

[2] T.G Bhaskar and V. Lakshmikantham, Fixed point theorems in partially oredered metric spaces and applications, Nonlinear Analysis:TMA, 65 (2006) 1379-1393.

[3] Berinde, V., Borcut M., Tripled fixed point theorems for contractive type mappings in partially ordered metric spaces, Nonlinear Anal., 74 (2011) 4889-4897.

[4] E. Karapinar, Couple Fixed Point on Cone Metric Space, Stud. Univ. Babes-Bolyai Math., 58 (2013) 75-85.

[5] L.G.Huang and X. Zhang, Cone metric spaces and fixed point theorems of contractive mapping, J. Math. Anal. Appl., 332 (2007) 1468-1476.

[6] M. Abbas, M. Ali Khan, S. Radenovic, Common coupled fixed point theorems in cone metric spaces for w-compatible mappings, Applied Mathematics and Computation, 217 (2010) 195-202.

[7] E. Karapinar, Coupled Fixed Point on Cone Metric Spaces, Gazi University Journal of Science, 1 (2011) 51-58.

[8] W. Long, B.E Rhoades and M. Rajovic, Copled Coincidence points for two mappings in metric spaces and cone metric spaces, Fixed Point Theory and Applications, 2012 (2012) $1-9$.

[9] Z. Kadelburg and S. Radenovic, Coupled Fixed point results under TVS-cone metric and w-cone-distance, Advances in Fixed point Theory, 2 (2012) 29-46.

[10] R. Batra and S.Vashistha, Coupled coincidence point theorems for nonlinear contractions under (F,g)-invariant set in cone metric spaces, J. Nonlinear Sci. Appl., 6((2013) 86-96.

[11] R. Batra, S. Vashistha and R. Kumar, Coupled coincidence point theorems for mappings without mixed monotone property under c-distance in cone metric spaces, J. Nonlinear Sci. Appl., 7 (2014) 345-358.

[12] E. Karapinar and D. Turkoglu, Best Approximations Theorems for a couple in Cone Banach Space, Fixed Point Theory and Applications, 2010 doi: 10.1155/2010/784578.

[13] W. Shantanawi, Some Common Coupled Fixed Point results in cone metric spaces, Int. Journal of Math. Analysis, 4 (2010) 2381-2388.

[14] A. Aghajani, M. Abbas and E. P. Kallehbasti, Coupled fixed point theorems in partially ordered metric spaces and application, Math. Commun., 17 (2012) 497-509.

[15] S. Sedghi, N. Shobkolaei,J. R. Roshan and W. Shatanawi, Coupled fixed point theorems G ${ }_{\mathrm{b}}$-Metric Space, MATEMATIQKI VESNIK, 2 (2014) 190-201.

[16] Jay G. Mehta and M. L. Joshi, On Coupled Fixed Point Theorem in Partially Ordered Complete Metric Space, Int. J. Pure Appl. Sci. Technol., 1 (2010) 87-92.

[17] N. Malhotra and B.Bansal, Some common coupled fixed point theorems for generalized contraction in b-metric space, J. Nonlinear Sci. Appl., 8 (2015) 8-16. 
